\title{
Rationale, Design, and Baseline Characteristics of Beijing Prediabetes Reversion Program: A Randomized Controlled Clinical Trial to Evaluate the Efficacy of Lifestyle Intervention and/or Pioglitazone in Reversion to Normal Glucose Tolerance in Prediabetes
}

\author{
Yingying Luo, ${ }^{1}$ Sanjoy K. Paul, ${ }^{2,3}$ Xianghai Zhou, ${ }^{1}$ Cuiqing Chang, ${ }^{4}$ Wei Chen, ${ }^{5}$ \\ Xiaohui Guo, ${ }^{6}$ Jinkui Yang, ${ }^{7}$ Linong Ji, ${ }^{1}$ and Hongyuan Wang ${ }^{8}$ \\ ${ }^{1}$ Department of Endocrinology and Metabolism, Peking University People's Hospital, Beijing, China \\ ${ }^{2}$ Melbourne EpiCentre, University of Melbourne, Melbourne, VIC, Australia \\ ${ }^{3}$ Clinical Trials and Biostatistics Unit, QIMR Berghofer Medical Research Institute, Brisbane, QLD, Australia \\ ${ }^{4}$ Institute of Sports Medicine, Peking University Third Hospital, Beijing, China \\ ${ }^{5}$ Department of Parenteral and Enteral Nutrition, Peking Union Medical College Hospital, Beijing, China \\ ${ }^{6}$ Department of Endocrinology and Metabolism, Peking University First Hospital, Beijing, China \\ ${ }^{7}$ Department of Endocrinology and Metabolism, Beijing Tongren Hospital, Capital Medical University, Beijing, China \\ ${ }^{8}$ Department of Epidemiology and Biostatistics, School of Public Health, Peking University Health Science Center, Beijing, China \\ Correspondence should be addressed to Linong Ji; jiln@bjmu.edu.cn and Hongyuan Wang; why_w2003@163.com
}

Received 24 October 2016; Accepted 28 November 2016; Published 12 January 2017

Academic Editor: Ruozhi Zhao

Copyright (C) 2017 Yingying Luo et al. This is an open access article distributed under the Creative Commons Attribution License, which permits unrestricted use, distribution, and reproduction in any medium, provided the original work is properly cited.

\begin{abstract}
Background. Patients with prediabetes are at high risk for diabetes and cardiovascular disease (CVD). No study has explored whether intervention could revert prediabetes to normal glycemic status as the primary outcome. Beijing Prediabetes Reversion Program (BPRP) would evaluate whether intensive lifestyle modification and/or pioglitazone could revert prediabetic state to normoglycemia and improve the risk factors of CVD as well. Methods. BPRP is a randomized, multicenter, $2 \times 2$ factorial design study. Participants diagnosed as prediabetes were randomized into four groups (conventional/intensive lifestyle intervention and $30 \mathrm{mg}$ pioglitazone/placebo) with a three-year follow-up. The primary endpoint was conversion into normal glucose tolerance. The trial would recruit 2000 participants (500 in each arm). Results. Between March 2007 and March 2011, 1945 participants were randomized. At baseline, the individuals were $53 \pm 10$ years old, with median BMI $26.0(23.9,28.2) \mathrm{kg} / \mathrm{m}^{2}$ and $\mathrm{HbA1c} 5.8$ (5.6, $6.1) \% .85 \%$ of the participants had IGT and $15 \%$ had IFG. Parameters relevant to glucose, lipids, blood pressure, lifestyle, and other metabolic markers were similar between conventional and intensive lifestyle intervention group at baseline. Conclusion. BPRP was the first study to determine if lifestyle modification and/or pioglitazone could revert prediabetic state to normoglycemia in Chinese population. Major baseline parameters were balanced between two lifestyle intervention groups. This trial is registered with www.chictr.org.cn: ChiCTR-PRC-06000005.
\end{abstract}

\section{Introduction}

About $6.9 \%$ of adults are estimated to have IGT (impaired glucose tolerance) globally, and the IGT prevalence is likely to be increased to $8 \%$ by 2035 [1]. The burden of prediabetes is significantly high in China. The national survey conducted during 2007-08 reported 15.5\% prevalence of prediabetes in adult Chinese population [2]. Patients with prediabetes are at high risk for both diabetes and its complications, especially the cardiovascular disease (CVD) $[3,4]$. Studies have shown that $1.5 \%-7.4 \%$ of individuals with prediabetes develop type 2 diabetes annually [4]. After 3-5 years of follow-up, 1/4 of 
the patients with prediabetes would develop type 2 diabetes [4]. The rate of incidence of diabetes in the control arm of $\mathrm{Da}$ Qing study was 15.7 per 1000 person years, during 6 years of follow-up [5]. Patients with prediabetes were also at high risk of developing CVD [6].

Over the last two decades, studies in China and other countries had shown that lifestyle intervention, with or without therapeutic intervention, could decrease the risk of developing type 2 diabetes in patients with prediabetes [5, 7-13]. In Da Qing study, after 6 years of follow-up, compared with control group, relative risk of developing type 2 diabetes was reduced by $42 \%$ in intensive lifestyle intervention group [5]. Finnish diabetes prevention study (DPS) compared the efficacies of lifestyle intervention in preventing diabetes in patients with prediabetes [8]. The incidence of diabetes in intervention group was less than half of that in control group after two years of follow-up. Diabetes prevention program (DPP) also showed that intensive lifestyle intervention in patients with prediabetes could prevent the development of type 2 diabetes in 1 of 7 followed up over 3 years [7]. Apart from lifestyle intervention, use of antidiabetes drugs (ADDs) had shown the effectiveness in preventing diabetes in patients with prediabetes [7, 10-14]. As compared with placebo, medication intervention might reduce the relative risk of developing diabetes by $25-60 \%$ and might increase the possibility of conversion rate up to $70 \%$ $[10,11,14-16]$.

Besides the prevention of diabetes, lifestyle intervention may also decrease the risk of CVD and mortality in prediabetes population. The 23-year follow-up data from the $\mathrm{Da}$ Qing study showed that the cumulative incidence of cardiovascular disease mortality was decreased from $19.6 \%$ to $11.9 \%$ in the lifestyle intervention group [17]. All-cause mortality was also decreased from $38.4 \%$ to $28.1 \%$ after an initial 6 years of lifestyle intervention [17]. Some studies also suggested that patients with prediabetes might also get some potential cardiovascular benefits from antidiabetic drugs since many surrogate markers were improved $[18,19]$.

Until now, three thiazolidinedione (TZD) drugs, including troglitazone, were used to prevent diabetes in IGT population. Troglitazone markedly reduced the incidence of diabetes during its limited period of use compared with all the other interventions in the DPP study [16]. Later on, rosiglitazone and pioglitazone were also tested to prevent diabetes in IGT population. Both of them showed a significant effect on reducing the risk of developing diabetes [4, 11, 12, 17]. However, no study have explored whether intervention could revert prediabetes to normal glycemic status as the primary outcome. The earlier studies were aimed at evaluating the efficacy of intervention on preventing progression to diabetes in individuals with impaired glucose tolerance (IGT), not prediabetes.

The purpose of Beijing Prediabetes Reversion Program (BPRP) was to examine whether lifestyle modification with or without pioglitazone could revert prediabetic state to normoglycemia over 3 years of follow-up in patients with prediabetes. Apart from the description of the study protocol of BPRP, the baseline characteristics of the randomized study subjects, by age groups and lifestyle intervention status, are also presented in this study.

\section{Materials and Method}

2.1. Study Design. Beijing Prediabetes Reversion Program (BPRP) is a prospective, multicenter, randomized, double blinded, and placebo controlled clinical trial, based on a $2 \times$ 2 factorial design. Patients with prediabetes were randomized into four groups: conventional lifestyle intervention + placebo, conventional lifestyle intervention + pioglitazone hydrochloride $30 \mathrm{mg}$ daily, intensive lifestyle intervention + placebo, and intensive lifestyle intervention + pioglitazone hydrochloride $30 \mathrm{mg}$ daily. The study hypothesis was that intensive lifestyle intervention and/or pioglitazone $30 \mathrm{mg}$ QD would increase the conversion rate of patients with prediabetes to normal glycemia, compared to conventional lifestyle intervention only.

Approval of protocol and consent forms by the local institutional review board was obtained at Peking University Health Science Center.

2.2. Trial Population. Individuals with high risk for diabetes were screened to confirm the glycemic state by oral glucose tolerance test (OGTT). High risk population included individuals with previously elevated fasting glucose level between 6.1 and $7.0 \mathrm{mmol} / \mathrm{L}$ or elevated 2-hour postprandial glucose level between 7.8 and $11.1 \mathrm{mmol} / \mathrm{L}$. Our goal was to recruit 2000 participants form outpatient departments at 36 public hospitals in Beijing, China. Patients with prediabetes (confirmed by OGTT) were eligible for inclusion. Major inclusion criteria are listed as follows.

\section{BPRP Study Major Inclusion Criteria}

(i) Voluntarily participating in the trial and signing subject's informed consent form

(ii) Prediabetic patients

(iii) Both males and females

(iv) Not limited to ethnicity

(v) 25 years of age-70 years of age

(vi) $22 \mathrm{~kg} / \mathrm{m}^{2} \leq \mathrm{BMI}<35 \mathrm{~kg} / \mathrm{m}^{2}$

Meanwhile, detailed inclusion and exclusion criteria are listed in Supplementary Table 1 (see Supplementary Material available online at https://doi.org/10.1155/2017/7602408). Informed consent was obtained before the individuals could participate in any screening procedures. Eligible participants were then randomized into one of the four arms of the study.

2.3. Randomization and Follow-Up. Randomization was undertaken by an independent statistician using a computer generated random sequence and was performed as block randomization with a 1:1:1:1 allocation ratio in four arms. Sealed envelopes were used for random allocations at the study sites. Both the participants and healthcare providers were blinded by the medication, while they were open to the lifestyle intervention. 


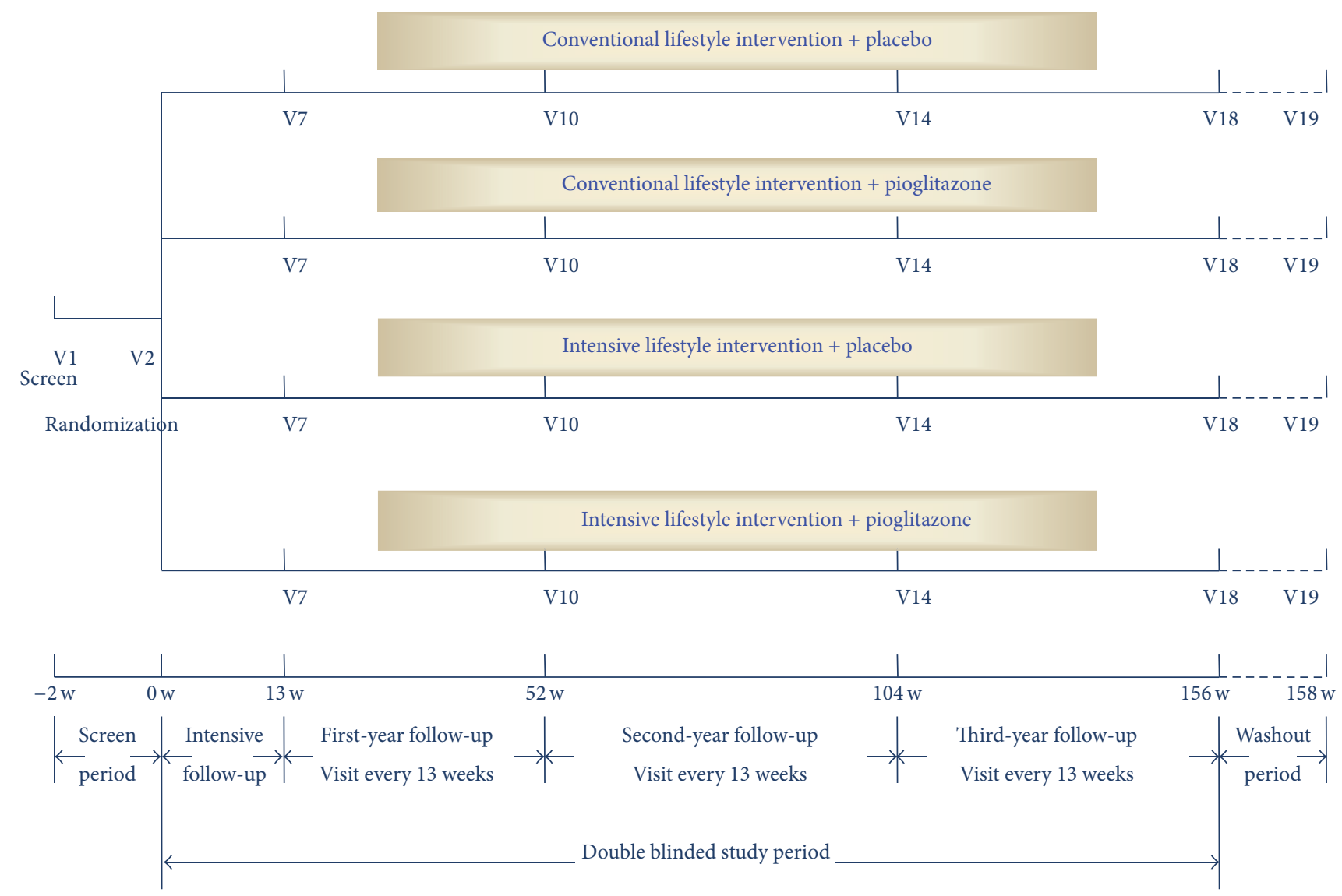

FIgURE 1: Study flow chart.

All the participants were recruited and followed up in the outpatient clinic in 36 study cites in Beijing. The followup of the study would last for three years with 19 scheduled visits. All participants would have an annual examination for glucose status (OGTT) during the follow-up period. Once the participants have been reverted to normal glucose state or have developed diabetes, defined by OGTT performed at annual examination, he or she would be terminated from the study (Figure 1).

For all four arms, study visits are scheduled for every 2-3 weeks during the first 13 weeks and every 13 weeks thereafter. Participants were required to complete a lifestyle diary comprising 3-day food records and average frequency of exercise per week for each visit.

In intensive lifestyle intervention group, participants would be educated at each visit after randomization, and the investigators would prescribe an individualized lifestyle prescription for them at each visit according to their body weight and lifestyle diary. A software program was developed to collect the lifestyle information from participants' lifestyle diary. Based on this information and the body weight at each visit, the software could calculate the compliance with lifestyle recommendations of each participant. The software would then generate a lifestyle prescription including the diet and exercise recommendations. Specific indicators of intensive lifestyle intervention are presented as follows.
Specific Indicators of Intensive Lifestyle Intervention

\section{Exercise Principle}

(i) Mainly whole-body aerobic endurance exercise, moderate intensity (3-6 MET), $\geq 30 \mathrm{~min} /$ day, 3-7 days/ week, $\geq 150 \mathrm{~min} /$ week, and $180-300 \mathrm{~min} /$ week, was recommended.

(ii) Resistance exercise (resisted movement) as supplement was as follows: $40-50 \%$ of 1 repetition maximum (40-50\% 1 RM), 3 sets of 8-10 exercises with 10-15 repetitions/set, and 2-3 days/week.

(iii) Moderate stretching exercise and flexibility exercise were as follows: $\geq 15 \mathrm{~min} /$ day and 3-7 days/week.

(iv) Energy consumption of exercise was as follows: total accumulative energy consumption $\geq 150 \mathrm{kcal} /$ day, typically $150-300 \mathrm{kcal} / \mathrm{day}$, and $\geq 750 \mathrm{kcal} / \mathrm{week}$, typically 900-1500 kcal/week.

(v) Exercise was performed according to three stages, that is, adaption stage, consolidation stage, and maintenance stage.

\section{Diet Principle}

(i) Based on Harris-Benedict formula [16], according to the participant's specific condition, required calorie 
was calculated, and rational diet plan was made for the participant.

\section{The Goal of Weight Control}

(i) For those with $\mathrm{BMI} \geq 24\left(\mathrm{~kg} / \mathrm{m}^{2}\right)$, waist circumference $\geq 80 \mathrm{~cm}$ in females, or waist circumference $\geq 85 \mathrm{~cm}$ in males, weight should be reduced according to negative energy balance principle.

(ii) The goal of weight loss was $5-10 \%$ of the current weight.

(iii) Weight loss rate was $2-4 \mathrm{~kg} /$ month.

In conventional lifestyle intervention group, participants would receive the usual lifestyle modification advice at baseline and at annual visits, without any individualized counseling. They would not get a lifestyle evaluation and lifestyle related prescription. To avoid the contamination between groups, similar visit schedule was designed for all the groups.

Among participants who received pioglitazone, the dose of the medication $(30 \mathrm{mg} /$ day) remains the same throughout the follow-up period. The active pioglitazone and the matched placebo were manufactured by Beijing Taiyang Pharmaceutical Company. The supply chain of active medication and placebo was managed by the study investigators at the participating study centers.

Participants who were identified to have achieved normal glucose level at annual visit were asked to stop the medication and were invited for OGTT two weeks after the last visit. This procedure was followed for those participants who remained prediabetic during the course of 3 years of followup. Those who remained prediabetic or regressed back to normal glucose status were advised to seek usual care. Those who were found to have developed diabetes after 2 weeks of washout period were also advised to seek standard care for diabetes. All participants were advised to follow standard lifestyle management at the end of follow-up.

2.4. Primary and Secondary Outcomes. The primary aim of the study was to evaluate the proportions of participants regressing back to normal glucose level during follow-up. The normal glucose level was defined as FPG $<6.1 \mathrm{mmol} / \mathrm{L}$ and $2 \mathrm{hPG}<7.8 \mathrm{mmol} / \mathrm{L}$ during the OGTT. The secondary outcomes of the study were as follows: (1) incidence of type 2 diabetes, (2) time to achieving normal glucose level, (3) change in HbAlc, (4) change in body weight and waist circumference, (5) changes in blood pressure, LDL-cholesterol, HDLcholesterol, and triglyceride, (6) changes in adiponectin, hsCRP, and insulin and C-peptide at fasting and after challenge, (7) change in urine albumin-creatinine ratio and serum creatinine, (8) composite of the incidence of at least one of the events-heart failure, nonfatal myocardial infarction, nonfatal stroke, or all-cause mortality, and (9) quality of life.

2.5. Study Measures. Details of study measurements are presented in Supplementary Table 2. At randomization and annual examinations, glucose tolerance status would be assessed by $75 \mathrm{~g}$ OGTT. OGTT was performed in the morning. All laboratory analyses are being conducted at the Peking
University Peoples Hospital's central laboratory. Data on physical activity and diet habits would be collected from patients' diary. All lifestyle data are fed into the software to calculate the total calorie intake and physical activity level. HbA1c was measured by HPLC (Ultra2 HbAlc Detector; Primus Corporation, Duluth, GA, USA; normal range 4$6 \%, 20-42 \mathrm{mmol} / \mathrm{mol})$. An immune-nephelometry method was used to measure the levels of LDL-cholesterol, HDLcholesterol, and triacylglycerol (COBAS Integra 400 Plus System; Roche Diagnostics, Basel, Switzerland). Insulin and C-peptide were measured by an electrochemiluminescence immunoassay (Elecsys 2010 system; Roche Diagnostics). All the study drugs were withheld in the morning of testing.

At 13 weeks from randomization, ALT, AST, and serum creatinine would be measured to monitor side effects of pioglitazone and to rule out the participants who have had serious conditions which may not be suitable for the continuation of the study. Vital signs, body weight, waist circumference, and blood pressure would be recorded at each study visit. Urine HCG would also be tested at each study visit in order to avoid the use of pioglitazone during unexpected pregnancy in women within gestational age. Participants who were found pregnant during follow-up were terminated from the study.

\subsection{Statistical Considerations}

2.6.1. Power Analysis. BPRP would recruit 2000 participants (500 in each arm) and would be followed for a planned maximum follow-up of 3 years. This sample size is expected to provide approximately $90 \%$ power with $5 \%$ type 1 error to detect $10 \%$ relative increase in the rate of primary outcomes among participants assigned to intensive lifestyle intervention compared with conventional lifestyle intervention group under the following assumptions:

(1) $35.3 \%$ for the conventional lifestyle plus placebo, $44.3 \%$ for the conventional lifestyle plus pioglitazone, $45.3 \%$ for the intensive lifestyle plus placebo, and $54.3 \%$ for the intensive lifestyle plus pioglitazone.

(2) Participants would be recruited in half a year.

(3) $30 \%$ of the participants might be lost to follow-up during the whole study.

2.6.2. Analysis Approach. The primary and secondary outcomes of the study will be evaluated following the intentionto-treat approach, with additional supporting analyses based on the per-protocol population. A separate Statistical Analysis Plan is in place which details the analysis approaches.

2.6.3. Statistical Methods for Baseline Data Analysis. The study participants were randomized at baseline into four groups. However, the distributions of baseline study parameters are presented by the intensive and conventional lifestyle group and by different categories of age at randomization. Basic statistics were presented by number (\%), mean (SD), or median (IQR) as appropriate. To evaluate the patterns of the distributions of glycemic parameters and body mass index by age groups, density plots were created. The distributions of 

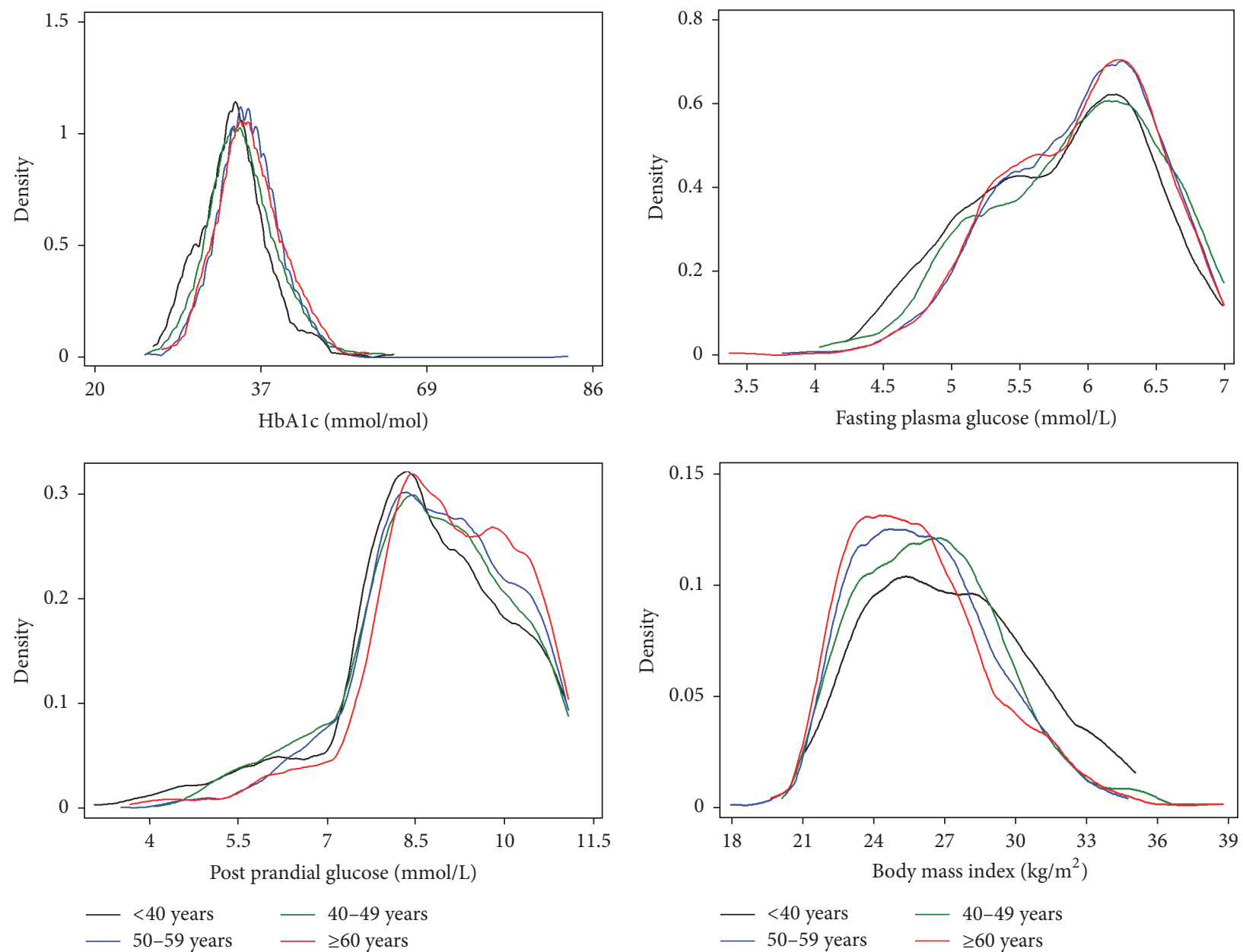

FIGURE 2: Density plots of $\mathrm{HbAlc}$, fasting plasma glucose, postprandial glucose, and body mass index by age categories at randomization.

the study parameters were not compared between the groups for possible differences.

\section{Results}

3.1. Patient Recruitment. From March 2007 to March 2011, 4397 individuals were screened who met the screening criteria. Among these individuals 2034 (46.3\%) were identified to have prediabetes. Following the inclusion and exclusion criteria, 1954 eligible patients were randomized to four groups in equal proportion in 36 participating study centers.

3.2. Baseline Characteristics. In the study cohort, $42 \%$ were male, with mean (SD) age 53 (10) years, median (IQR) BMI $26.0(23.9,28.2) \mathrm{kg} / \mathrm{m}^{2}, 49 \%$ were overweight and $12 \%$ were obese and $23 \%$ were current or ex-smokers (Table 1). Older patients were significantly less likely to be current or exsmokers and obese, compared to patients below the age of 40 years (Table 2). Of all the participants, only $24 \%$ had low level physical activity.

The distributions of fasting and postprandial plasma glucose levels were similar between lifestyle intervention groups and across the age groups at randomization (Tables 1 and 2, Figure 2). With an average HbAlc level of $5.3 \%$
(34 $\mathrm{mmol} / \mathrm{mol})$ at baseline, about $7 \%$ patients had HbAlc $\geq$ $6.5 \%$ ( $48 \mathrm{mmol} / \mathrm{mol})$. About $15 \%$ participants were identified with isolated IFG, while most of the subjects (85\%) had IGT (54\% had isolated IGT and $31 \%$ had IFG plus IGT). The distribution of metabolic and other risk factors were similar across age groups.

\section{Discussion}

BPRP is the first study to determine whether lifestyle modification and/or pioglitazone could revert prediabetic state back to normoglycemia in Chinese population and to explore the mechanism through which different interventions exert their effects on glucose metabolism and cardiovascular risk factors. Compared with previous diabetes prevention studies, our study has several unique features. First, while most of the earlier studies evaluated the efficacy of different interventions to prevent the development of diabetes in individuals with IGT, our study aims at evaluating the efficacy of intensive lifestyle intervention with or without TZD to regress back the prediabetic individuals to normoglycemic status $[5,10$, 13, 20-23]. Only few studies have examined the effect of intervention(s) on conversion into normoglycemia in individuals with prediabetes [12]. However, the regression back 
TABLE 1: Characteristics of participants by different lifestyle intervention at randomization.

\begin{tabular}{|c|c|c|c|}
\hline & Conventional & Intensive & All \\
\hline$N$ & $972(50)$ & $973(50)$ & 1945 \\
\hline \multicolumn{4}{|l|}{ Sex } \\
\hline Male & $450(46)$ & $374(38)$ & $824(42)$ \\
\hline \multicolumn{4}{|l|}{ Age (years) } \\
\hline mean $\pm \mathrm{SD}$ & $52 \pm 10$ & $53 \pm 10$ & $53 \pm 10$ \\
\hline \multicolumn{4}{|l|}{ Occupation } \\
\hline Professional/business & $447(47)$ & $427(45)$ & $874(46)$ \\
\hline Workers & $59(6)$ & $53(6)$ & $112(6)$ \\
\hline Retired & $362(38)$ & $396(42)$ & $758(40)$ \\
\hline Jobless/other & $83(9)$ & $76(8)$ & $159(8)$ \\
\hline \multicolumn{4}{|l|}{ Ethnicity } \\
\hline Han & $934(97)$ & $927(96)$ & $1861(96)$ \\
\hline Others & $31(3)$ & $41(4)$ & $72(4)$ \\
\hline \multicolumn{4}{|l|}{ Education level } \\
\hline Low & $46(5)$ & $42(4)$ & $88(5)$ \\
\hline Middle & $468(49)$ & $449(47)$ & $917(48)$ \\
\hline High & $436(46)$ & $468(49)$ & $904(47)$ \\
\hline \multicolumn{4}{|l|}{ Smoking state } \\
\hline Current smoker or past smoker & $234(24)$ & $202(21)$ & $436(23)$ \\
\hline \multicolumn{4}{|l|}{ Body shape } \\
\hline $\operatorname{BMI}\left(\mathrm{kg} / \mathrm{m}^{2}\right)$ & $26(24,28)$ & $26(24,28)$ & $26(24,28)$ \\
\hline Normal: BMI $<25$ & $385(40)$ & $360(37)$ & $745(38)$ \\
\hline Overweight: $25 \leq \mathrm{BMI}<30$ & $464(48)$ & $495(51)$ & $959(49)$ \\
\hline Obese: $\mathrm{BMI} \geq 30$ & $123(13)$ & $118(12)$ & $241(12)$ \\
\hline Waist $(\mathrm{cm})$ & $89(83,96)$ & $88(82,95)$ & $89(82,95)$ \\
\hline \multicolumn{4}{|l|}{ Blood pressure } \\
\hline Systolic blood pressure $(\mathrm{mmHg})$ & $120(111,130)$ & $120(110,130)$ & $120(110,130)$ \\
\hline Diastolic blood pressure $(\mathrm{mmHg})$ & $79(70,81)$ & $77(70,80)$ & $78(70,80)$ \\
\hline \multicolumn{4}{|l|}{ Glucose level } \\
\hline Fasting plasma glucose $(\mathrm{mmol} / \mathrm{L})$ & $6.0(5.5,6.4)$ & $6.0(5.5,6.4)$ & $6.0(5.5,6.4)$ \\
\hline $2 \mathrm{~h}$ plasma glucose $(\mathrm{mmol} / \mathrm{L})$ & $8.8(8.1,9.8)$ & $8.9(8.1,9.9)$ & $8.9(8.1,9.9)$ \\
\hline HbAlc (\%) & $5.8(5.6,6.0)$ & $5.8(5.5,6.1)$ & $5.8(5.6,6.1)$ \\
\hline $\mathrm{HbAlc}(\mathrm{mmol} / \mathrm{mol})$ & $40(38,42)$ & $40(37,43)$ & $40(38,43)$ \\
\hline $\mathrm{HbAlc}<5.7 \%(39 \mathrm{mmol} / \mathrm{mol})$ & $331(34)$ & $327(34)$ & $658(34)$ \\
\hline $5.7 \%(39 \mathrm{mmol} / \mathrm{mol}) \leq \mathrm{HbAlc}<6.5 \%(48 \mathrm{mmol} / \mathrm{mol})$ & $572(59)$ & $572(59)$ & $1144(59)$ \\
\hline $\mathrm{HbAlc} \geq 6.5 \%(48 \mathrm{mmol} / \mathrm{mol})$ & $69(7)$ & $74(8)$ & $143(7)$ \\
\hline IGT & $821(85)$ & $833(86)$ & $1654(85)$ \\
\hline Isolated IGT & $532(55)$ & $525(54)$ & $1057(54)$ \\
\hline $\mathrm{IFG}+\mathrm{IGT}$ & $289(30)$ & $308(32)$ & $597(31)$ \\
\hline Isolated IFG & $151(16)$ & $140(14)$ & $291(15)$ \\
\hline \multicolumn{4}{|l|}{ Lipid level } \\
\hline Total cholesterol (mmol/L) & $4.9(4.3,5.5)$ & $4.9(4.3,5.4)$ & $4.9(4.3,5.5)$ \\
\hline $\mathrm{LDL}-\mathrm{C}(\mathrm{mmol} / \mathrm{L})$ & $3.2(2.7,3.7)$ & $3.1(2.6,3.7)$ & $3.2(2.6,3.7)$ \\
\hline $\mathrm{HDL}-\mathrm{C}(\mathrm{mmol} / \mathrm{L})$ & $1.2(1.0,1.4)$ & $1.2(1.0,1.4)$ & $1.2(1.0,1.4)$ \\
\hline Triglyceride $(\mathrm{mmol} / \mathrm{L})$ & $1.5(1.1,2.1)$ & $1.5(1.1,2.0)$ & $1.5(1.1,2.1)$ \\
\hline \multicolumn{4}{|l|}{ Liver function } \\
\hline ALT (U/L) & $21(16,31)$ & $21(15,29)$ & $21(16,30)$ \\
\hline AST (U/L) & $22(18,26)$ & $21(17,26)$ & $21(18,26)$ \\
\hline \multicolumn{4}{|l|}{ Hemoglobin } \\
\hline Hemoglobin $(\mathrm{g} / \mathrm{L})$ & $143(134,153)$ & $141(132,152)$ & $142(133,153)$ \\
\hline
\end{tabular}


TABLE 1: Continued.

\begin{tabular}{|c|c|c|c|}
\hline & Conventional & Intensive & All \\
\hline \multicolumn{4}{|l|}{ HOMA } \\
\hline HOMA-IR & $2.4(1.6,3.5)$ & $2.4(1.6,3.6)$ & $2.4(1.6,3.5)$ \\
\hline HOMA-beta & $77.5(50.8,113.1)$ & $79.9(52.3,116.7)$ & $78.5(51.8,114.7)$ \\
\hline \multicolumn{4}{|l|}{ Cytokines } \\
\hline $\mathrm{CRP}(\mu \mathrm{mol} / \mathrm{L})$ & $1.2(0.7,2.3)$ & $1.2(0.7,2.4)$ & $1.2(0.7,2.4)$ \\
\hline Adiponectin & $6.2(4.2,8.8)$ & $6.2(4.3,9.0)$ & $6.2(4.3,8.9)$ \\
\hline SOD & $6.8(4.1,10.3)$ & $6.9(4.3,10.5)$ & $6.9(4.2,10.4)$ \\
\hline Amylin & $7.7(6.5,9.5)$ & $7.5(6.4,9.3)$ & $7.6(6.4,9.4)$ \\
\hline IL-6 & $2.3(1.5,4.2)$ & $2.3(1.5,3.8)$ & $2.3(1.5,4.0)$ \\
\hline \multicolumn{4}{|l|}{ Urine ACR } \\
\hline Urine albumin/Cr (mg/g) & $7.4(4.5,15.2)$ & $7.4(4.4,14.3)$ & $7.4(4.5,14.7)$ \\
\hline \multicolumn{4}{|l|}{ Diet } \\
\hline Daily calories intake (kcal/d) & $1521(1242,1874)$ & $1554(1248,1920)$ & $1535(1243,1899)$ \\
\hline Proportion of total calories intake from carbohydrate (\%) & $60(51,69)$ & $59(50,67)$ & $60(50,68)$ \\
\hline Proportion of total calories intake from protein (\%) & $14(12,16)$ & $14(12,17)$ & $14(12,17)$ \\
\hline Proportion of total calories intake from fat (\%) & $23(17,30)$ & $24(18,31)$ & $24(17,30)$ \\
\hline \multicolumn{4}{|l|}{ Physical activity } \\
\hline Low level & $232(25)$ & $224(24)$ & $456(24)$ \\
\hline Medium level & $483(52)$ & $501(53)$ & $984(53)$ \\
\hline High level & $223(24)$ & $213(23)$ & $436(23)$ \\
\hline
\end{tabular}

Note. Estimates for continuous study parameters are presented by median (IQR), unless otherwise stated. Categorical study parameters are presented by number (percentage).

to normoglycemia was not the primary outcome of these studies.

In individuals with IGT, previous studies have showed that intensive lifestyle intervention can reduce the incidence of diabetes by $31 \%-58 \%[5,15,24]$. The goal of lifestyle intervention, however, is difficult to achieve and maintain. Treatment of IGT with oral antidiabetic drugs, such as metformin, acarbose, or TZDs, has been shown to prevent or delay progression to diabetes in high risk individuals [10-12, 15] or prior gestational diabetes mellitus [25]. In addition, TZDs have shown greater efficacy in preventing IGT developing to diabetes, compared to that observed with acarbose or metformin. In IGT individuals receiving TZDs, the relative risk was reduced by $55-72 \%[11,12,25]$, compared to a risk reduction of $31 \%$ and $25 \%$ in IGT individuals receiving metformin [15] and acarbose [10], respectively.

Individuals with prediabetes receiving rosiglitazone were more likely to regress to normoglycemia compared with individuals receiving placebo [11]. After 5.7 years of median follow-up in DPP study, individuals who returned to normoglycemia at least once had a reduced risk of developing diabetes compared with individuals who consistently had prediabetes [26]. Increased $\beta$-cell function and insulin sensitivity may contribute to the reduced risk for diabetes in individuals who returned to normoglycemia during the intervention [26]. This suggests individuals who returned to normoglycemia may benefit more in terms of preventing diabetes. Studies aimed at evaluating the effect of intervention on conversion into normoglycemia in individuals with prediabetes and exploring the possible mechanisms involved in the conversion are needed.

Secondly, our prediabetic study population included both isolated elevated IFG and IGT population. Most of the earlier studies, including Da Qing study, DPP study, DPS study and ACT NOW trial, evaluated only the IGT population $[5,20,21$, 23]. The mechanism of isolated elevated fasting glucose level may be different with that of elevated postprandial glucose level. Only DREAM trial included individuals with IGT and with isolated IFG [22]. However, the primary outcome of this study was the incidence of diabetes during follow-up, and the efficacy of intensive lifestyle intervention was not evaluated with the intervention therapy (rosiglitazone and/or ramipril). Also, there is no data in Chinese population with isolated IFG. Our study would provide new insight into the possible efficacy of combination of lifestyle intervention and TZD in individuals with isolated IFG.

Thirdly, with a baseline BMI of $26 \mathrm{~kg} / \mathrm{m}^{2}$, our study offered an excellent opportunity to evaluate the possible efficacy of intensive lifestyle intervention with or without intervention with $\mathrm{ADD}$ in normal weight and overweight individuals. The Da Qing study and the Indian Prevention Program $[5,13]$ had similar BMI in the study population. However, the primary outcomes and the interventions in these studies were different. Other studies, primarily based on Caucasians from Europe and America, show a higher BMI level at baseline (around $30 \mathrm{~kg} / \mathrm{m}^{2}$ ) [12, 20, 22, 23]. However, our participants were not that obese as Caucasians in most previous diabetes prevention studies. So the goal we have set for intensive lifestyle intervention group might be a little bit difficult to achieve. But this may also provide us with an opportunity to find a proper goal of lifestyle intervention among normal weight population with prediabetes in the future.

Lastly, as there has been rapid development in the Chinese society and its lifestyle over the last decade, the general population is receiving more and more information from 
TABLE 2: Characteristics of participants by different age groups at randomization.

\begin{tabular}{|c|c|c|c|c|}
\hline & $<40$ years old & $40-49 y$ & $50-59 y$ & $\geq 60 y$ \\
\hline \multicolumn{5}{|l|}{ Total } \\
\hline$N$ & $237(12)$ & $462(234)$ & $802(41)$ & $444(23)$ \\
\hline \multicolumn{5}{|l|}{ Sex } \\
\hline Male & $138(58)$ & $238(52)$ & $272(34)$ & $176(40)$ \\
\hline \multicolumn{5}{|l|}{ Occupation } \\
\hline Professional/business & $185(80)$ & $330(73)$ & $313(40)$ & $46(11)$ \\
\hline Workers & $17(7)$ & $35(8)$ & $46(6)$ & $14(3)$ \\
\hline Retired & $0(0)$ & $37(8)$ & $367(47)$ & $354(82)$ \\
\hline Jobless/others & $30(13)$ & $48(11)$ & $62(8)$ & $19(4)$ \\
\hline \multicolumn{5}{|l|}{ Ethnicity } \\
\hline Han & $226(96)$ & $446(97)$ & $775(97)$ & $414(94)$ \\
\hline Others & $9(4)$ & $16(4)$ & $22(3)$ & $25(6)$ \\
\hline \multicolumn{5}{|l|}{ Education level } \\
\hline Low & $1(0)$ & $12(3)$ & $37(5)$ & $38(9)$ \\
\hline Middle & $63(27)$ & $194(43)$ & $454(58)$ & $206(48)$ \\
\hline High & $170(73)$ & $250(55)$ & $299(38)$ & $185(43)$ \\
\hline \multicolumn{5}{|l|}{ Smoking state } \\
\hline Smoker (current or past) & $76(32)$ & $143(31)$ & $145(18)$ & $72(16)$ \\
\hline \multicolumn{5}{|l|}{ Body shape } \\
\hline BMI $\left(\mathrm{kg} / \mathrm{m}^{2}\right)$ & $27(25,30)$ & $26(24,28)$ & $26(24,28)$ & $25(24,28)$ \\
\hline Normal: BMI $<25$ & $73(31)$ & $164(36)$ & $316(39)$ & $192(43)$ \\
\hline Overweight: $25 \leq \mathrm{BMI}<30$ & $111(47)$ & $246(53)$ & $398(50)$ & $204(46)$ \\
\hline Obese: $\mathrm{BMI} \geq 30$ & $53(22)$ & $52(11)$ & $88(11)$ & $48(11)$ \\
\hline Waist $(\mathrm{cm})$ & $90(84,98)$ & $90(83,96)$ & $88(82,94)$ & $88(83,95)$ \\
\hline \multicolumn{5}{|l|}{ Blood pressure } \\
\hline Systolic blood pressure $(\mathrm{mmHg})$ & $118(110,123)$ & $120(110,126)$ & $120(112,130)$ & $125(119,133)$ \\
\hline Diastolic blood pressure $(\mathrm{mmHg})$ & $76(70,80)$ & $78(70,81)$ & $79(70,81)$ & $78(70,80)$ \\
\hline \multicolumn{5}{|l|}{ Glucose level } \\
\hline Fasting plasma glucose $(\mathrm{mmol} / \mathrm{L})$ & $5.9(5.3,6.3)$ & $6.0(5.4,6.4)$ & $6.0(5.5,6.4)$ & $6.0(5.5,6.3)$ \\
\hline $2 \mathrm{~h}$ plasma glucose $(\mathrm{mmol} / \mathrm{L})$ & $8.7(7.9,9.6)$ & $8.8(8.0,9.7)$ & $9.0(8.1,9.9)$ & $9.0(8.3,10.0)$ \\
\hline HbAlc (\%) & $5.7(5.4,5.9)$ & $5.7(5.5,6.0)$ & $5.8(5.6,6.1)$ & $5.8(5.6,6.1)$ \\
\hline $\mathrm{HbAlc}(\mathrm{mmol} / \mathrm{mol})$ & $39(36,41)$ & $39(37,42)$ & $40(38,43)$ & $40(38,43)$ \\
\hline $\mathrm{HbAlc}<5.7 \%(39 \mathrm{mmol} / \mathrm{mol})$ & $114(48)$ & $180(39)$ & $237(30)$ & $127(29)$ \\
\hline $5.7 \%(39 \mathrm{mmol} / \mathrm{mol}) \leq \mathrm{HbAlc}<6.5 \%(48 \mathrm{mmol} / \mathrm{mol})$ & $112(47)$ & $248(54)$ & $507(63)$ & $277(62)$ \\
\hline $\mathrm{HbAlc} \geq 6.5 \%(48 \mathrm{mmol} / \mathrm{mol})$ & $11(5)$ & $34(7)$ & $58(7)$ & $40(9)$ \\
\hline IGT & $198(84)$ & $379(82)$ & $680(85)$ & $397(89)$ \\
\hline Isolated IGT & $142(60)$ & $253(55)$ & $425(53)$ & $237(53)$ \\
\hline $\mathrm{IFG}+\mathrm{IGT}$ & $56(24)$ & $126(27)$ & $255(32)$ & $160(36)$ \\
\hline Isolated IFG & $39(17)$ & $83(18)$ & $122(15)$ & $47(11)$ \\
\hline \multicolumn{5}{|l|}{ Lipid level } \\
\hline Total cholesterol $(\mathrm{mmol} / \mathrm{L})$ & $4.6(4.0,5.2)$ & $4.8(4.2,5.3)$ & $5.0(4.3,5.7)$ & $4.9(4.3,5.5)$ \\
\hline $\mathrm{LDL}-\mathrm{C}(\mathrm{mmol} / \mathrm{L})$ & $2.9(2.5,3.4)$ & $3.1(2.6,3.6)$ & $3.3(2.7,3.8)$ & $3.2(2.7,3.7)$ \\
\hline HDL-C (mmol/L) & $1.1(1.0,1.3)$ & $1.2(1.0,1.3)$ & $1.2(1.0,1.4)$ & $1.3(1.1,1.5)$ \\
\hline Triglyceride (mmol/L) & $1.7(1.2,2.5)$ & $1.5(1.1,2.1)$ & $1.5(1.1,2.1)$ & $1.4(1.1,1.9)$ \\
\hline \multicolumn{5}{|l|}{ Liver function } \\
\hline $\operatorname{ALT}(\mathrm{U} / \mathrm{L})$ & $26(18,43)$ & $22(16,32)$ & $21(16,29)$ & $18(14,24)$ \\
\hline AST (U/L) & $22(18,28)$ & $20(17,26)$ & $22(18,26)$ & $21(18,25)$ \\
\hline
\end{tabular}


TABLE 2: Continued.

\begin{tabular}{|c|c|c|c|c|}
\hline & $<40$ years old & $40-49 y$ & $50-59 y$ & $\geq 60 y$ \\
\hline \multicolumn{5}{|l|}{ Hemoglobin } \\
\hline Hemoglobin $(\mathrm{g} / \mathrm{L})$ & $148(135,161)$ & $146(134,157)$ & $140(133,150)$ & $140(131,148)$ \\
\hline \multicolumn{5}{|l|}{ HOMA } \\
\hline HOMA-IR & $3.2(1.8,4.2)$ & $2.5(1.5,3.4)$ & $2.4(1.7,3.5)$ & $2.3(1.5,3.3)$ \\
\hline HOMA-beta & $103.8(66.0,157.1)$ & $80.1(51.9,115.8)$ & $75.4(51.5,109.9)$ & $73.4(48.7,104.1)$ \\
\hline \multicolumn{5}{|l|}{ Cytokines } \\
\hline $\mathrm{CRP}(\mu \mathrm{mol} / \mathrm{L})$ & $1.1(0.6,2.3)$ & $1.1(0.7,2.4)$ & $1.2(0.7,2.4)$ & $1.3(0.7,2.4)$ \\
\hline Adiponectin & $5.2(3.7,7.0)$ & $5.6(3.7,8.2)$ & $6.3(4.6,9.3)$ & $6.9(4.7,9.5)$ \\
\hline SOD & $6.5(3.7,9.7)$ & $7.0(4.3,10.4)$ & $6.9(4.1,10.8)$ & $6.7(4.5,10.0)$ \\
\hline Amylin & $7.7(6.6,9.4)$ & $7.6(6.5,9.5)$ & $7.5(6.4,9.3)$ & $7.5(6.3,9.7)$ \\
\hline IL-6 & $2.2(1.5,4.2)$ & $2.0(1.5,3.4)$ & $2.4(1.5,4.1)$ & $2.5(1.6,4.3)$ \\
\hline \multicolumn{5}{|l|}{ Urine ACR } \\
\hline Urine albumin/Cr (mg/g) & $6.4(4.1,13.5)$ & $7.4(4.3,14.3)$ & $7.4(4.5,14.5)$ & $7.7(4.8,16.9)$ \\
\hline \multicolumn{5}{|l|}{ Diet } \\
\hline Daily calories intake (kcal/d) & $1619(1259,2032)$ & $1557(1230,1946)$ & $1512(1254,1841)$ & $1515(1244,1893)$ \\
\hline Proportion of total calories intake from carbohydrate (\%) & $58(50,66)$ & $59(49,68)$ & $59(50,68)$ & $61(53,68)$ \\
\hline Proportion of total calories intake from protein (\%) & $14(12,17)$ & $15(12,17)$ & $14(12,17)$ & $14(12,16)$ \\
\hline Proportion of total calories intake from fat (\%) & $25(19,31)$ & $24(17,31)$ & $24(18,30)$ & $23(16,29)$ \\
\hline \multicolumn{5}{|l|}{ Physical activity } \\
\hline Low level & $95(42)$ & $133(30)$ & $168(22)$ & $60(14)$ \\
\hline Medium level & $108(47)$ & $241(54)$ & $411(53)$ & $224(53)$ \\
\hline High level & $25(11)$ & $75(17)$ & $195(25)$ & $141(33)$ \\
\hline
\end{tabular}

Note. Estimates for continuous study parameters are presented by median (IQR), unless otherwise stated. Categorical study parameters are presented by number (percentage).

media on how to prevent diabetes. Therefore, in such an era of information explosion, it remains unknown whether individuals receiving intensive lifestyle intervention would at all show any significant benefit over those in the control group. Our study would provide necessary information to answer this question. To maximize the potential benefits from intensive lifestyle intervention, individualized lifestyle education and computerized prescription would be given to the participants in intensive lifestyle treatment group. This individualized education is based on the characteristics of each patient, considered with their body weight, diet habit, and exercise preference.

In summary, BPRP addresses the dramatically increasing population of prediabetes in China which is a major public health problem. A possible positive effect of the intensive lifestyle intervention on conversion rate from prediabetes into normoglycemia would provide a simple and powerful public health message. On the other hand, a finding that this intervention had no effect or was detrimental would be equally important and would indicate that efforts to improve diabetes care should be directed elsewhere.

\section{Conclusion}

BPRP was the first study to determine if lifestyle modification and/or pioglitazone could revert prediabetic state to normoglycemia in Chinese population. Major baseline parameters were balanced between two lifestyle intervention groups.
In addition, with a baseline BMI of $26 \mathrm{~kg} / \mathrm{m}^{2}$, our study also offers an excellent opportunity to evaluate the possible efficacy of intensive lifestyle intervention with or without intervention with antidiabetic drug in normal weight and overweight individuals. Our study addresses the dramatically increasing population of prediabetes in China which is a major public health problem. This randomized clinical trial would provide the evidence of whether intensive lifestyle intervention and/or pioglitazone might convert prediabetes back into normoglycemia and would also quantify the benefits of the conversion into normoglycemia in different intervention groups.

\section{Abbreviations}

$\begin{array}{ll}\text { ACR: } & \text { Albumin/creatinine } \\ \text { ADD: } & \text { Antidiabetes drug } \\ \text { BMI: } & \text { Body mass index } \\ \text { BPRP: } & \text { Beijing Prediabetes Reversion Program } \\ \text { CRP: } & \text { C-reactive protein } \\ \text { CVD: } & \text { Cardiovascular disease } \\ \text { DPP: } & \text { Diabetes prevention program } \\ \text { DPS: } & \text { Finnish diabetes prevention study } \\ \text { HbAlc: } & \text { Hemoglobin Alc } \\ \text { HDL-C: } & \text { HDL-cholesterol }\end{array}$

HOMA-beta: Homeostatic model assessment for beta cell function 
HOMA-IR: Homeostatic model assessment for insulin resistance

IFG: Impaired fasting glucose

IGT: Impaired glucose tolerance

IL-6: Interleukin-6

LDL-C: LDL-cholesterol

OGTT: Oral glucose tolerance test

PG: $\quad$ Plasma glucose

SOD: $\quad$ Superoxide dismutase

TC: Total cholesterol

TG: $\quad$ Triglyceride

TZD: Thiazolidinedione.

\section{Ethical Approval}

Approval of protocol and consent forms by the local institutional review board was obtained at Peking University Health Science Center.

\section{Consent}

Informed consent was obtained before the individuals could participate in any screening procedures.

\section{Disclosure}

Infrastructure research support from the Australian Government's National Collaborative Research Infrastructure Strategy (NCRIS) initiative through Therapeutic Innovation Australia had no role in the design of this study and would not have any role during its execution, analyses, interpretation of the data, or decision to submit results. Beijing Taiyang Pharmaceutical Company has given all the medication support in this trial, without participating in study design, drug choice, execution, data analysis, and reporting.

\section{Competing Interests}

Sanjoy K. Paul has acted as a consultant and speaker for Novartis, GI Dynamics, and Amylin Pharmaceuticals LLC. He has received grants in support of investigator and investigator initiated clinical studies from Merck, Novo Nordisk, Hospira, AstraZeneca, Amylin Pharmaceuticals, and Pfizer. All the other authors declare that there is no conflict of interests regarding the publication of this paper.

\section{Authors' Contributions}

Linong Ji, Hongyuan Wang, Yingying Luo, Xianghai Zhou, Cuiqing Chang, and Wei Chen were responsible for the conceptualization and design of the Trial. Yingying Luo, Xianghai Zhou, Xiaohui Guo, and Jinkui Yang were involved in data acquisition. Sanjoy K. Paul and Hongyuan Wang performed the analysis and interpretation of the data. Yingying Luo, Sanjoy K. Paul, Xianghai Zhou, and Linong Ji drafted the manuscript. Cuiqing Chang, Wei Chen, Xiaohui Guo, Jinkui Yang, and Hongyuan Wang revised the manuscript for critical intellectual content. All authors approved the final the final version of the manuscript. Yingying Luo, Sanjoy K. Paul, Hongyuan Wang, and Linong Ji had full access to all the data in the study and take responsibility for the integrity of the data and the accuracy of the data analysis. Yingying Luo and Sanjoy K. Paul contributed equally to this work and should be considered co-first authors.

\section{Acknowledgments}

Funding support was received from the Beijing Municipal Science \& Technology Commission (Grant nos. D0905003040131, D131100005313008, and D121100004412002). QIMR Berghofer gratefully acknowledges infrastructure research support from the Australian Government's National Collaborative Research Infrastructure Strategy (NCRIS) initiative through Therapeutic Innovation Australia. The authors thank Beijing Taiyang Pharmaceutical Company to have given all the medication support in this trial. They thank all the participants for participating in this study. The authors also acknowledge all the principle investigators in the following study sites for their contribution to this trial: Beijing Tongren Hospital: Jingkui Yang; Beijing Hospital: Lixin Guo; The Military General Hospital of Beijing PLA: Xiaofeng Lv; Peking University First Hospital: Xiaohui Guo; Peking Union Medical Colledge Hospital: Hongding Xiang; Fuxing Hospital: Xiaoming Zhuang; Beijing No. 6 Hospital: Shangnong Wang; The Second Artillery General Hospital of Chinese People's Liberation Army: Quanmin Li; Beijing Zhanlanlu Hospital: Shuling Chang; Peking University Third Hospital: Tianpei Hong; Chinese PLA General Hospital: Juming Lu; The 304th Hospital: Shinan Yin; The 309th Hospital of Chinese People's Liberation Army: Yan Zhang; Navy General Hospital: Qiyu Guo; Air Force General Hospital: Xiaohong Guan; Beijing Haidian Hospital: Wei Huang; China-Japan Friendship Hospital: Guangwei Li; The 306th Hospital of PLA: Zhangrong Xu; Beijing Chaoyang Hospital: Yuan Xu; Beijing Chuiyangliu Hospital: Cuiping Liu; China Meitan General Hospital: Hongmei Li; Civil Aviation General Hospital: Dingqiong Peng; Xuanwu Hospital: Li Wang; Beijing Tiantan Hospital: Lirong Zhong; Aerospace 731 Hospital: Yuming Liu; Beijing Electric Power Hospital: Dongmei Ni; The Luhe Teaching Hospital of the Capital Medical University: Dong Zhao; People's Hospital of Beijing Daxing District: Changchun Xue; Beijing Pinggu Hospital: Yufeng Li; Daxing Hospital of Tranditional Chinese Medicine: Li Ma; Beijing Chaoyang Hospital (Jingxi Campus): Shan Gao; Beijing Hepingli Hospital: Yaping Liu; Peking University Shougang Hospital: Xiaoping Lu; Nanyuan Hospital: Kaijie Yang; Beijiao Hospital: Z. Jing; Nanfaxin Hospital: Rongmin Zhang.

\section{References}

[1] International Diabetes Federation, IDF Diabetes Atlas, 2013.

[2] W. Yang, J. Lu, J. Weng et al., "Prevalence of diabetes among men and women in China," The New England Journal of Medicine, vol. 362, no. 12, pp. 1090-1101, 2010.

[3] S. M. Grundy, "Pre-diabetes, metabolic syndrome, and cardiovascular risk," Journal of the American College of Cardiology, vol. 59, no. 7, pp. 635-643, 2012. 
[4] M. I. Harris, "impaired glucose tolerance-prevalence and conversion to NIDDM," Diabetic Medicine, vol. 13, no. 2, pp. S9-S11, 1996.

[5] X.-R. Pan, G.-W. Li, Y.-H. Hu et al., "Effects of diet and exercise in preventing NIDDM in people with impaired glucose tolerance. The Da Qing IGT and diabetes study," Diabetes Care, vol. 20, no. 4, pp. 537-544, 1997.

[6] R. P. Donahue and T. J. Orchard, "Diabetes mellitus and macrovascular complications. An epidemiological perspective," Diabetes Care, vol. 15, no. 9, pp. 1141-1155, 1992.

[7] W. C. Knowler, S. E. Fowler, R. F. Hamman et al., "10-Year follow-up of diabetes incidence and weight loss in the Diabetes Prevention Program Outcomes Study," The Lancet, vol. 374, pp. 1677-1686, 2009.

[8] J. Lindström, A. Louheranta, M. Mannelin et al., "The Finnish Diabetes Prevention Study (DPS): lifestyle intervention and 3year results on diet and physical activity," Diabetes Care, vol. 26, no. 12, pp. 3230-3236, 2003.

[9] J. Lindström, P. Ilanne-Parikka, M. Peltonen et al., "Sustained reduction in the incidence of type 2 diabetes by lifestyle intervention: follow-up of the Finnish Diabetes Prevention Study," The Lancet, vol. 368, no. 9548, pp. 1673-1679, 2006.

[10] J.-L. Chiasson, R. G. Josse, R. Gomis, M. Hanefeld, A. Karasik, and M. Laakso, "Acarbose for prevention of type 2 diabetes mellitus: the STOP-NIDDM randomised trial," The Lancet, vol. 359, no. 9323, pp. 2072-2077, 2002.

[11] H. C. Gerstein, S. Yusuf, J. Bosch et al., "Effect of rosiglitazone on the frequency of diabetes in patients with impaired glucose tolerance or impaired fasting glucose: a randomised controlled trial," The Lancet, vol. 368, no. 9541, pp. 1096-1105, 2006.

[12] R. A. DeFronzo, D. Tripathy, D. C. Schwenke et al., "Pioglitazone for diabetes prevention in impaired glucose tolerance," The New England Journal of Medicine, vol. 364, no. 12, pp. 1104-1115, 2011.

[13] A. Ramachandran, C. Snehalatha, S. Mary, B. Mukesh, A. D. Bhaskar, and V. Vijay, "The Indian Diabetes Prevention Programme shows that lifestyle modification and metformin prevent type 2 diabetes in Asian Indian subjects with impaired glucose tolerance (IDPP-1)," Diabetologia, vol. 49, no. 2, pp. 289297, 2006.

[14] J. S. Torgerson, J. Hauptman, M. N. Boldrin, and L. Sjöström, "XENical in the Prevention of Diabetes in Obese Subjects (XENDOS) study: a randomized study of orlistat as an adjunct to lifestyle changes for the prevention of type 2 diabetes in obese patients," Diabetes Care, vol. 27, no. 1, pp. 155-161, 2004.

[15] W. C. Knowler, E. Barrett-Connor, S. E. Fowler et al., "Reduction in the incidence of type 2 diabetes with lifestyle intervention or metformin," The New England Journal of Medicine, vol. 346, no. 6, pp. 393-403, 2002.

[16] W. C. Knowler, R. F. Hamman, S. L. Edelstein et al., "Prevention of type 2 diabetes with troglitazone in the Diabetes Prevention Program," Diabetes, vol. 54, no. 4, pp. 1150-1156, 2005.

[17] G. Li, P. Zhang, J. Wang et al., "Cardiovascular mortality, allcause mortality, and diabetes incidence after lifestyle intervention for people with impaired glucose tolerance in the Da Qing Diabetes Prevention Study: a 23-year follow-up study," The Lancet Diabetes and Endocrinology, vol. 2, no. 6, pp. 474-480, 2014.

[18] R. Ratner, R. Goldberg, S. Haffner et al., "Impact of intensive lifestyle and metformin therapy on cardiovascular disease risk factors in the diabetes prevention program," Diabetes Care, vol. 28, no. 4, pp. 888-894, 2005.
[19] M. Hanefeld, J. L. Chiasson, C. Koehler, E. Henkel, F. Schaper, and T. Temelkova-Kurktschiev, "Acarbose slows progression of intima-media thickness of the carotid arteries in subjects with impaired glucose tolerance," Stroke, vol. 35, no. 5, pp. 1073-1078, 2004.

[20] J. Eriksson, J. Lindström, T. Valle et al., "Prevention of Type II diabetes in subjects with impaired glucose tolerance: the Diabetes Prevention Study (DPS) in Finland. Study design and 1year interim report on the feasibility of the lifestyle intervention programme," Diabetologia, vol. 42, no. 7, pp. 793-801, 1999.

[21] R. A. Defronzo, M. A. Banerji, G. A. Bray et al., "Actos Now for the prevention of diabetes (ACT NOW) study," BMC Endocrine Disorders, vol. 9, article 17, 2009.

[22] H. C. Gerstein, S. Yusuf, R. Holman, J. Bosch, and J. Pogue, "Rationale, design and recruitment characteristics of a large, simple international trial of diabetes prevention: the DREAM trial," Diabetologia, vol. 47, no. 9, pp. 1519-1527, 2004.

[23] The Diabetes Prevention Program Research Group, “The Diabetes Prevention Program: baseline characteristics of the randomized cohort," Diabetes Care, vol. 23, pp. 1619-1629, 2000.

[24] J. Tuomilehto, J. Lindström, J. G. Eriksson et al., "Prevention of type 2 diabetes mellitus by changes in lifestyle among subjects with impaired glucose tolerance," The New England Journal of Medicine, vol. 344, no. 18, pp. 1343-1350, 2001.

[25] T. A. Buchanan, A. H. Xiang, R. K. Peters et al., "Preservation of pancreatic $\beta$-cell function and prevention of type 2 diabetes by pharmacological treatment of insulin resistance in high-risk Hispanic women," Diabetes, vol. 51, no. 9, pp. 2796-2803, 2002.

[26] L. Perreault, Q. Pan, K. J. Mather, K. E. Watson, R. F. Hamman, and S. E. Kahn, "Effect of regression from prediabetes to normal glucose regulation on long-term reduction in diabetes risk: results from the Diabetes Prevention Program Outcomes Study," The Lancet, vol. 379, no. 9833, pp. 2243-2251, 2012. 


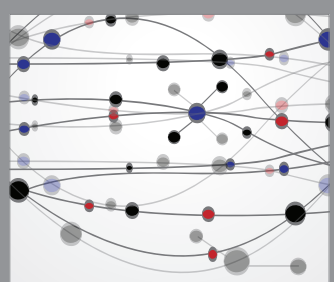

The Scientific World Journal
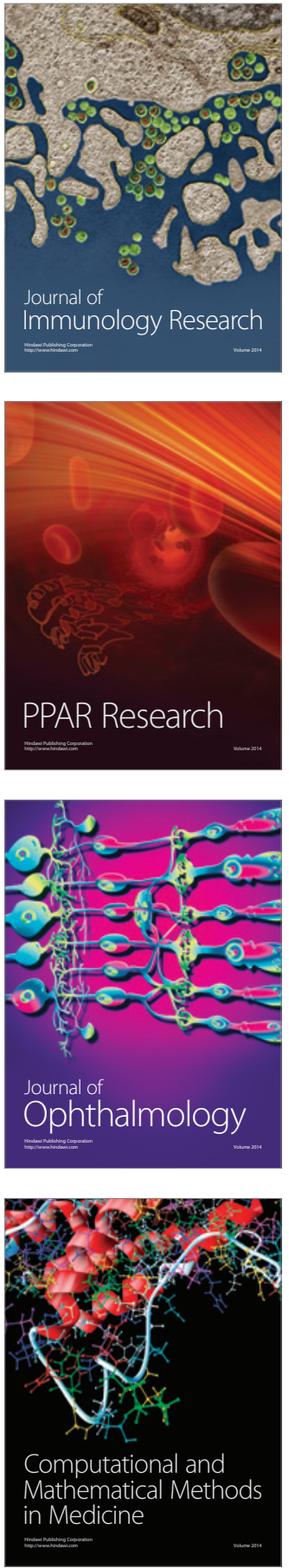

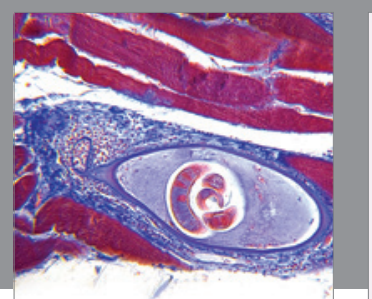

Gastroenterology Research and Practice
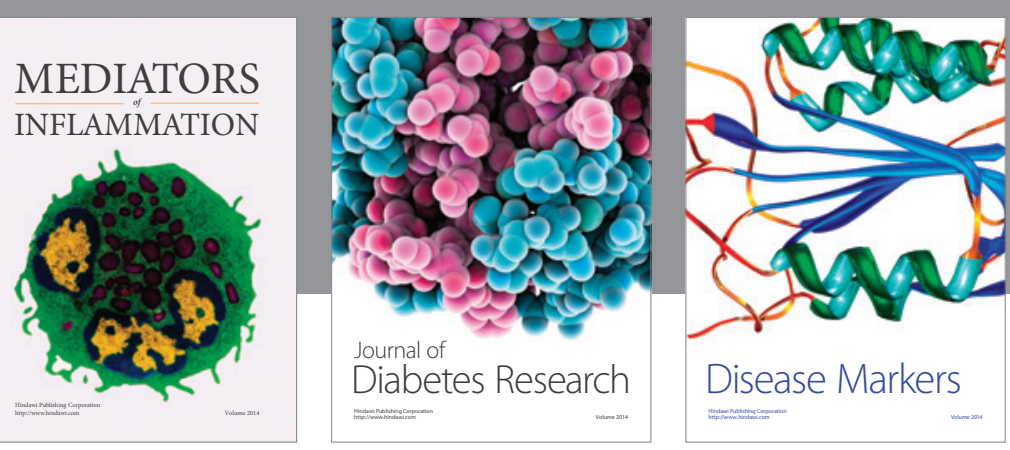

Disease Markers

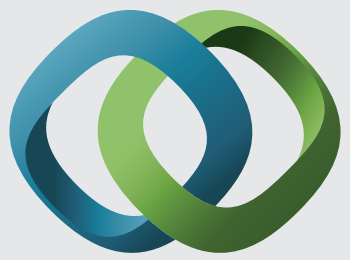

\section{Hindawi}

Submit your manuscripts at

https://www.hindawi.com
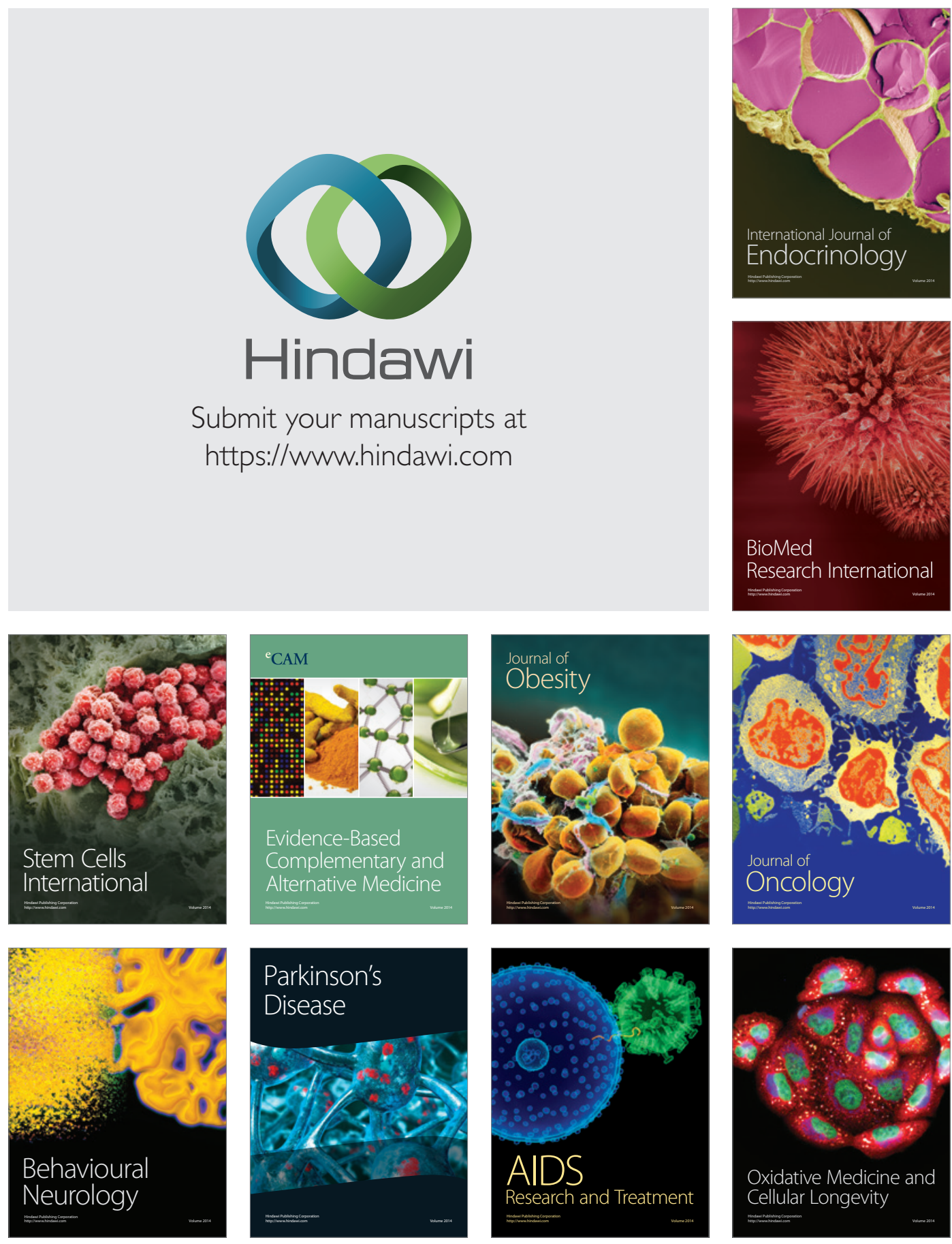